\title{
Influence of Primary or Secondary Muscle Invasive Bladder Cancer on Oncologic Outcomes After Radical Cystectomy
}

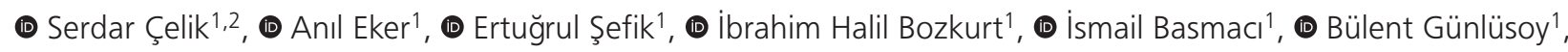 \\ (D) Tansu Değirmenci ${ }^{1}$ \\ ${ }^{1}$ University of Health Sciences Turkey, Izmir Bozyaka Training and Research Hospital, Clinic of Urology, Izmir, Turkey \\ ${ }^{2}$ Dokuz Eylül University, Institute of Oncology, Department of Basic Oncology, Izmir, Turkey
}

\begin{abstract}
Objective: In this study, we aimed to investigate the oncologic outcomes of patients who were diagnosed with primary (muscle invasive cancer at the time of diagnosis) and secondary (cancer that showed progression from non-muscle invasive bladder cancer) muscle invasive bladder carcinoma (MIBC) before radical cystectomy (RC).

Materials and Methods: Patients who underwent RC at our clinic for MIBC between July 2008 and June 2017 were included in the study. They were divided into two groups based on their diagnosis as primary and secondary MIBC. Their clinical, pathological, and oncologic data (upstaging, adjuvant chemotherapy, overall mortality, overall survival, cancer-specific mortality, and cancer-specific survival) were evaluated retrospectively.

Results: A total of 80 patients underwent RC due to bladder cancer with a mean age of $64.4 \pm 7.8$ years (range: $42-83$ years) and a mean follow-up time of $32.9 \pm 32.1$ months (range: 1-113 months). The overall and cancer-specific survivals of the patients were $64.7 \pm 6.6$ and $74 \pm 6.8$ months, respectively. Sixty-five and 15 patients were evaluated in the primary and secondary MIBC groups, respectively. Lymph node metastasis was higher in primary MIBC group ( $p=0.031$ ). Although, there were no statistical differences between the groups, in secondary MIBC group, the overall survival $(67.3 \pm 7.2$ months vs $42.5 \pm 8.4$ months; $p=0.835)$ and cancerspecific survival ( $77.6 \pm 7.4$ months vs $46.6 \pm 8.6$ months; $p=0.546)$ were lower, while the overall mortality $(44.6 \%$ vs $53.3 \% ; p=0.372)$ and cancer-specific mortality (32.3\% vs $46.7 \% ; p=0.293$ ) were higher compared to primary MIBC group.

Conclusion: The pre- and postoperative pathological T stages were similar between the groups, whereas postoperative lymph node positivity was lower for secondary MIBC patients. The mortality was higher and overall and cancer-specific survivals were lower in secondary MIBC patients; however, these findings were not statistically significant.
\end{abstract}

Keywords: Bladder cancer, muscle invasive bladder cancer, oncologic outcomes, radical cystectomy, secondary muscle invasive bladder cancer

\section{Introduction}

According to the latest statistics, bladder cancer is the fourth most common cancer in men and the eighth in mortality (1). Approximately $75 \%$ of patients are non-muscle invasive bladder cancer (NMIBC), while $25 \%$ are muscle invasive bladder cancer (MIBC) at the time of diagnosis $(2,3)$. The best treatment method for MIBC is extended lymph node dissection and radical cystectomy (RC) with urinary diversion. In the NMIBC group, especially in high risk patients, in addition to conservative treatment, RC can also be recommended due to the $30-50 \%$ risk of progression to MIBC (4-6). When we look at the MIBC patients we come across two groups of patients. These are patients who have been previously diagnosed as having highrisk NMIBC and have progressed to MIBC with muscle tissue invasion in their follow-up (secondary MIBC), and those who have been diagnosed as having primary MIBC at the time of initial diagnosis, and the best treatment modality is RC for both two groups $(6,7)$. The requirement for chemotherapy (CT) before RC (neoadjuvant) or after RC (adjuvant) varies according to patient characteristics and physician's selection (8). When we look at the RC series, it is seen that $57 \%$ of the patients treated

Cite this article as: Çelik S, Eker A, Şefik E, Bozkurt iH, Basmacı I, Günlüsoy B, Değirmenci T. Influence of Primary or Secondary Muscle Invasive Bladder Cancer on Oncologic Outcomes After Radical Cystectomy. Bull Urooncol 2020;19(4):186-190

Address for Correspondence: Serdar Çelik, University of Health Sciences Turkey, İzmir Bozyaka Training and Research Hospital, Clinic of Urology, Dokuz Eylül University, Institute of Oncology, Department of Basic Oncology, Izmir, Turkey Phone: +90 5057019631 E-mail: serdarcelik84@hotmail.com ORCID-ID: orcid.org/0000-0003-0939-9989 
with RC due to primary MIBC and the rest are secondary MIBC (9). However, the oncological results of these two groups of patients are still discussed in the literature with conflicting results (7). For this reason, in this article, we investigated the post-RC oncological results of patients with primary and secondary MIBC who were diagnosed as having MIBC before RC.

\section{Materials and Methods}

Patients who underwent RC in our clinic due to MIBC between July 2008 and June 2017 were retrospectively analyzed. The study was retrospective and ethics committee approval was not applied for. Patients who had complete data and were followed up in our clinic and diagnosed as having MIBC (urothelial carcinoma) after transurethral resection (TURBT) were included in the study. Patients with non-urothelial bladder cancer, upper urinary tract tumor, NMIBC (Tis, Ta and T1), clinical T4 tumors, and missing follow-up data were not included in the study. The general characteristics, preoperative, peroperative and postoperative data of the patients were reviewed. General clinical characteristics of the patients (age, gender, American Society of Anesthesiologists score, Eastern Cooperative Oncology Group performance score, Charlson comorbidity index, presence of hydronephrosis), peroperative data (operation time, urinary diversion preference), pathological data (preoperative T stage and, grade, presence of carcinoma in situ (CIS), presence of squamous differentiation, postoperative $T$ stage and grade, surgical margin positivity, lymph node positivity, number of dissected lymph nodes number and percentage of positive nodes, nodes, prostate, urethra and ureter invasions, lymphovascular invasion and perineural invasion) and oncological outcomes (upstaging, adjuvant CT, overall mortality and survival time, cancer-specific mortality and survival time) were evaluated. The patients were divided into two groups as primary and secondary MIBC. Patients with muscle invasion at the time of diagnosis [patients having muscle invasion according to the pathology report of the first TUR-BT ( \pm re-TUR)] were included in the primary MIBC group. In the secondary MIBC group, patients who were followed up due to NMIBC and whose muscle invasion was detected in the last TUR-BT pathology were included.

\section{Statistical Analysis}

The data were analyzed using the Statistical Package for Social Sciences, version 20.0 (SPSS, Chicago, III) program. The primary and secondary MIBC groups were compared using the MannWhitney $U$ and chi-square tests. Additionally, Kaplan-Maier survival analysis and log-rank test were used for overall survival and cancer-specific survival data. The data in the tables were given as mean \pm standard deviation, and the statistical results were analyzed over the median data. Statistical significance was accepted as p-value $<0.05$.

\section{Results}

In our clinic, among 167 patients who underwent RC for urothelial carcinoma of bladder 80 patients, all of whom were followed up in our clinic and who had complete data, were evaluated. Eight $(10 \%)$ of the patients were female. The mean age was $64.4 \pm 7.8$
(42-83) years and the mean follow-up period was 32.9 \pm 32.1 (1113) months. The mean overall and cancer-specific survivals of the patients were $64.7 \pm 6.6$ and $74 \pm 6.8$ months. Sixty five and 15 patients were evaluated in primary and secondary MIBC groups, respectively. Patient characteristics and preoperative data and comparison results of patients with primary and secondary MIBC are given in Table 1. In the comparison between groups, patient characteristics and preoperative data were found to be similar. Preoperative, peroperative and postoperative pathological findings and comparison results of the groups are given in Table 2. Among the pathological data, lymph node metastasis was found to be higher in the primary MIBC group $(p=0.031)$. While the number of dissected lymph nodes was similar between the groups $(p=0.726)$, the number and percentage of positive lymph nodes were higher in the primary MIBC group $(p=0.033$ and $p=0.041$ ). Other pathological findings, urinary diversion preference and adjuvant CT status, and oncological results were similar between the groups (Tables 2 and 3). In primary MIBC group, Overall survival (67.3 \pm 7.2 months for primary

Table 1. Patient characteristics, preoperative findings and comparison results in primary and secondary muscle invasive bladder carcinoma groups

\begin{tabular}{|c|c|c|c|c|}
\hline & & $\begin{array}{l}\text { Primary } \\
\text { MIBC } \\
(n=65)\end{array}$ & $\begin{array}{l}\text { Secondary } \\
\text { MIBC } \\
(n=15)\end{array}$ & $\mathbf{p}$ \\
\hline \multicolumn{2}{|l|}{$\begin{array}{l}\text { Age (years), } \\
\text { mean } \pm \text { SD (min-max) }\end{array}$} & $\begin{array}{l}63.8 \pm 8.3 \\
(42-83)\end{array}$ & $\begin{array}{l}67.2 \pm 4 \\
(60-74)\end{array}$ & 0.081 \\
\hline \multirow{2}{*}{ Gender, n (\%) } & Female & $5(7.7)$ & $3(20)$ & \multirow{2}{*}{0.166} \\
\hline & Male & $60(92.3)$ & $12(80)$ & \\
\hline \multicolumn{2}{|l|}{$\begin{array}{l}\text { TUR number, } \\
\text { mean } \pm S D \text { (min-max) }\end{array}$} & $\begin{array}{l}1 \text { (re-TUR in } \\
5 \text { patients) }\end{array}$ & $\begin{array}{l}2.9 \pm 1.1 \\
(2-6)\end{array}$ & - \\
\hline \multicolumn{2}{|l|}{$\begin{array}{l}\text { Follow-up time, } \\
\text { mean } \pm S D(\text { min-max })\end{array}$} & $\begin{array}{l}33.2 \pm 33.8 \\
(0.3-119.6)\end{array}$ & $\begin{array}{l}31.6 \pm 24.8 \\
(2.1-77.8)\end{array}$ & 0.618 \\
\hline \multirow{4}{*}{ ASA score, n (\%) } & 1 & 5 & 0 & \multirow{4}{*}{0.145} \\
\hline & 2 & 43 & 9 & \\
\hline & 3 & 16 & 5 & \\
\hline & 4 & 1 & 1 & \\
\hline \multirow{5}{*}{$\begin{array}{l}\text { ECOG performance } \\
\text { score, } \mathrm{n}(\%)\end{array}$} & 0 & 17 & 3 & \multirow{5}{*}{0.199} \\
\hline & 1 & 35 & 5 & \\
\hline & 2 & 11 & 5 & \\
\hline & 3 & 2 & 2 & \\
\hline & 4 & 0 & 0 & \\
\hline \multirow{4}{*}{$\begin{array}{l}\text { Charlson comorbidity } \\
\text { index, } \mathrm{n}(\%)\end{array}$} & 0 & 1 & 0 & \multirow{4}{*}{0.147} \\
\hline & 1 & 3 & 0 & \\
\hline & 2 & 14 & 0 & \\
\hline & $3+$ & 47 & 15 & \\
\hline \multicolumn{2}{|l|}{$\begin{array}{l}\text { Preoperative } \\
\text { hydronephrosis, n (\%) }\end{array}$} & $27(41.5)$ & $4(26.7)$ & 0.210 \\
\hline \multirow{2}{*}{ Hydronephrosis, n (\%) } & Unilateral & $22(92.3)$ & $3(20)$ & \multirow{2}{*}{0.598} \\
\hline & Bilateral & $5(7.7)$ & $1(6.7)$ & \\
\hline \multicolumn{5}{|c|}{$\begin{array}{l}\text { Mann-Whitney U test, chi-square test and Fisher's Exact test. SD: Standarc } \\
\text { deviation, MIBC: Muscle invasive bladder carcinoma, ASA: American Society o } \\
\text { Anesthesiologists, ECOG: Eastern Cooperative Oncology Group, min: Minimum } \\
\text { max: Maximum }\end{array}$} \\
\hline
\end{tabular}




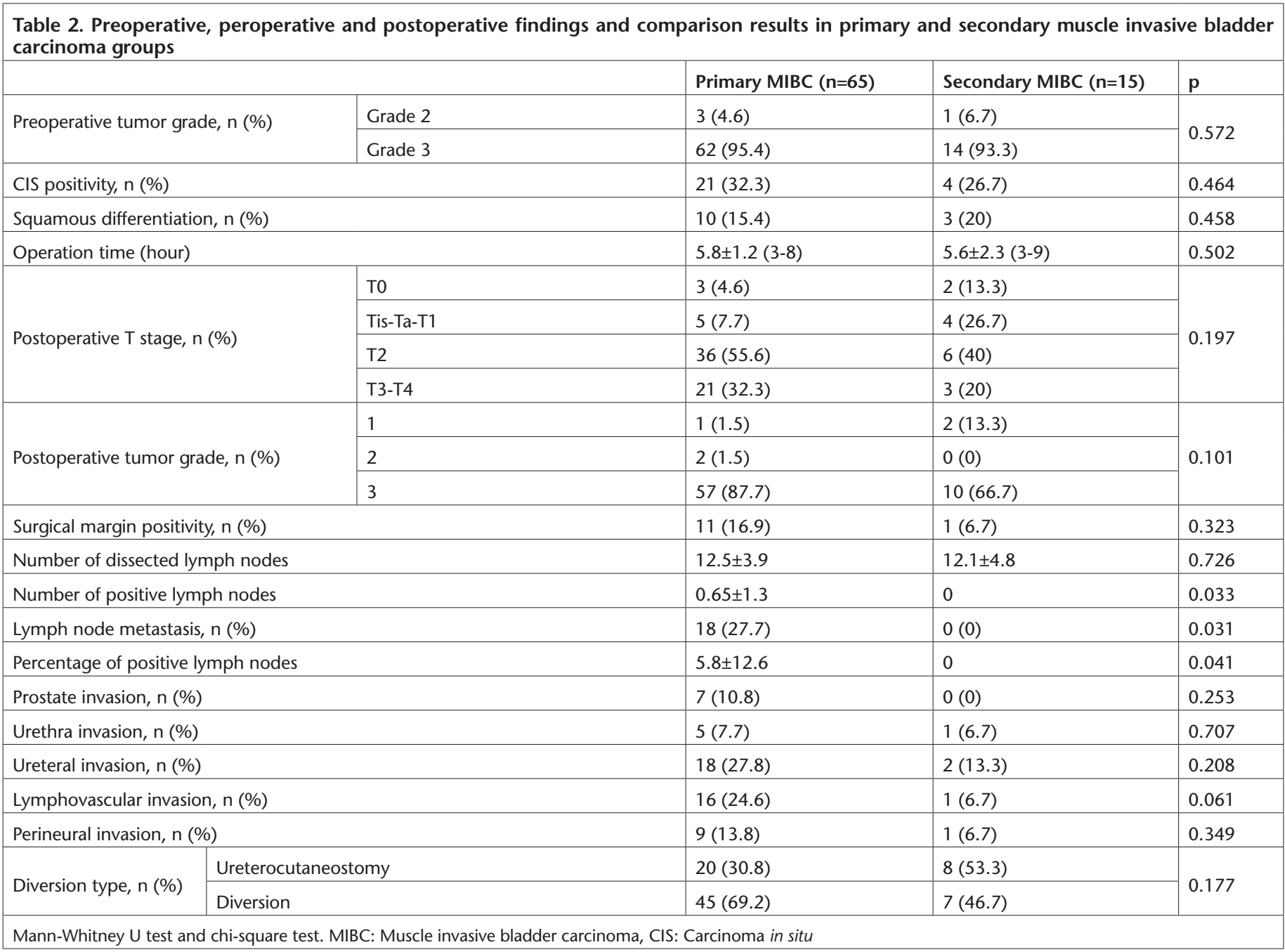

MIBC and 42.5 \pm 8.4 months for secondary MIBC, $p=0.835)$ and cancer specific survival (77.6 \pm 7.4 months for primary MIBC and 46.6 \pm 8.6 months for secondary MIBC, $p=0.546$ ) were higher and mortality rates (overall mortality $44.6 \%$ vs $53.3 \%, p=0.372$; cancer specific mortality $32.3 \%$ vs $46.7 \%, p=0.293$ ) were lower than secondary MIBC group, but there was no statistically significant difference between the two groups. The overall and cancer specific survival curves of the patients are given in Figure 1 and Figure 2.

\section{Discussion}

In comparisons between the primary and secondary MIBC groups; pathological T stage, tumor grade, surgical margin positivity and the number of dissected lymph nodes were similar between the groups, while number of lymph node positivity and percentage of lymph node positivity were higher in the primary MIBC group. When we looked at the oncological results, although there was no statistically significant difference, the numerical differences between the groups were remarkable. Accordingly, although the rates of T2-4 N + patients and adjuvant CT were higher in the primary MIBC group; the overall survival (67.3 \pm 7.2 months vs $42.5 \pm 8.4$ months; $p=0.835$ ) and cancer-specific
Table 3. Postoperative findings, oncological results and comparison results of primary and secondary muscle invasive bladder carcinoma groups

\begin{tabular}{|c|c|c|c|c|}
\hline & & $\begin{array}{l}\text { Primary } \\
\text { MIBC } \\
(n=65)\end{array}$ & $\begin{array}{l}\text { Secondary } \\
\text { MIBC } \\
(n=15)\end{array}$ & p \\
\hline \multicolumn{2}{|l|}{ Up-staging, n (\%) } & $22(33.8)$ & $4(26.7)$ & 0.418 \\
\hline \multirow{3}{*}{ Stage change, n (\%) } & Up-staging & $22(33.8)$ & $4(26.7)$ & \multirow{3}{*}{0.138} \\
\hline & Down-staging & $8(12.3)$ & $5(33.3)$ & \\
\hline & No change & $35(53.8)$ & $6(40)$ & \\
\hline \multicolumn{2}{|c|}{ Adjuvant chemotherapy, n (\%) } & $16(24.6)$ & $1(6.7)$ & 0.114 \\
\hline \multicolumn{2}{|l|}{ Overall mortality, n (\%) } & $29(44.6)$ & $8(53.3)$ & 0.372 \\
\hline \multicolumn{2}{|l|}{ Overall survival } & $67.3 \pm 7.2$ & $42.5 \pm 8.4$ & 0.835 \\
\hline \multicolumn{2}{|c|}{ Cancer specific mortality, n (\%) } & $21(32.3)$ & $7(46.7)$ & 0.293 \\
\hline \multicolumn{2}{|l|}{ Cancer specific survival } & $77.6 \pm 7.4$ & $46.6 \pm 8.6$ & 0.546 \\
\hline \multicolumn{2}{|l|}{ Length of stay } & $11.7 \pm 5.6$ & $11.8 \pm 6.7$ & 0.482 \\
\hline
\end{tabular}




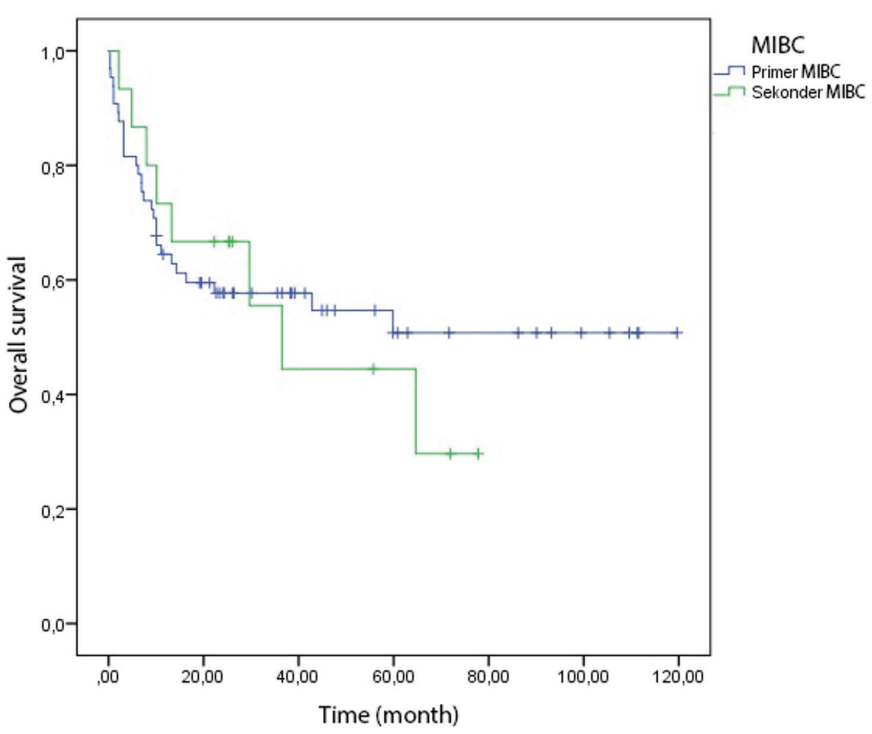

Figure 1. Overall survival curves of primary and secondary muscle invasive bladder carcinoma groups

MIBC: Muscle invasive bladder carcinoma

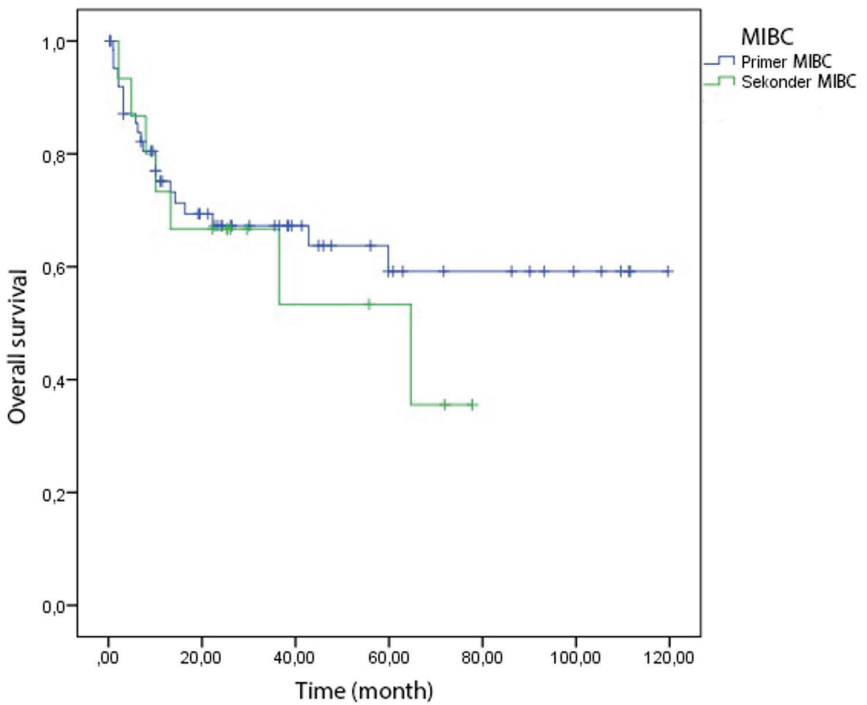

Figure 2. Cancer specific survival curves of primary and secondary muscle invasive bladder carcinoma groups

MIBC: Muscle invasive bladder carcinoma

survival (77.6 \pm 7.4 months vs $46.6 \pm 8.6$ months; $\mathrm{p}=0.546)$ were lower, overall mortality ( $44.6 \%$ vs $53.3 \% ; \mathrm{p}=0.372$ ) and cancer specific mortality ( $32.3 \%$ vs $46.7 \% ; p=0.293$ ) were higher in the secondary MIBC group without reaching a statistical significance.

In a study conducted by Moschini et al. (10) with 768 patients with primary and secondary (progressive) MIBC, 10-year recurrence-free survival ( $43 \%$ vs $36 \% ; p=0.01$ ), cancer specific survival ( $43 \%$ vs $37 \% ; p=0.01$ ) and overall mortality ( $35 \%$ vs $28 \% ; p=0.03$ ) results were shown to be worse in the secondary MIBC group. On the other hand, in a study by Aziz et al. (11), cancer-specific survival was similar in primary and secondary
MIBC, while subgroup analysis of secondary MIBC showed that high European Organization for Research and Treatment of Cancer (EORTC) score was associated with short cancer-specific survival. In that study, it was emphasized that the EORTC risk score was not only associated with the progression to MIBC but also with the results after RC and the importance of early RC in these patients was highlighted (11). In a recently published meta-analysis evaluating 13 important studies, it was reported that oncologic results were worse in patients with secondary MIBC compared to patients with primary MIBC (7). Although early RC was emphasized in patients with secondary MIBC, it was also emphasized that it could not be routinely recommended in all patients with NMIBC (7). Considering the heterogeneity and progression-related factors (high grade, pT1 stage, multifocality, tumor diameter $(>3 \mathrm{~cm}), \mathrm{CIS}$, complete non-responsiveness and P53 expression) in addition to the organ-sparing approach was important in disease management $(7,12,13)$. Therefore, it can be concluded that oncological results may be poor after diagnosis of MIBC especially in patients with high risk of progression and that early RC is an important option in these patients.

Many studies have examined the time until RC after MIBC diagnosis and it has been shown that oncological results worsen as the time gets longer $(14,15,16)$. It is also one of the questions to be asked that whether the time until RC in patients with secondary MIBC may be similarly prolonged. When the time until RC was examined in a study, it was observed that $12.5 \%$ of patients with primary MIBC had a period of $>3$ months, whereas this rate was $28.7 \%$ in patients with secondary MIBC (17). When we looked at the factors that may have caused this delay, neoadjuvant $\mathrm{CT}$ appeared as a reason. As a matter of fact, in a recent study by Pietzak et al. (18), it was shown that secondary MIBC was more resistant to neoadjuvant CT. In addition, another reason that may have prolonged this period, according to us, was that the patients became accustomed to the disease and wanted to avoid an operation with high morbidity such as RC. Therefore, it should be emphasized that the patient management should be discussed in detail with this patient group and that especially the oncological results may deteriorate. When we returned to the study conducted by Pietzak et al. (18), we saw that there were genomic differences between primary and secondary MIBC. In that study, 288 patients (245 patients with primary MIBC and 43 with secondary MIBC) who received neoadjuvant CT out of 2105 patients who underwent RC have been evaluated. Mortality developed in 103 of the patients with a median follow-up of 4 years. When the pathological responses were examined, it has been observed that the neoadjuvant chemo-response was lower in the secondary MIBC group compared to the primary MIBC group ( $45 \%$ vs $26 \%$ ). At the same time, recurrence-free survival, cancer-specific survival and overall survival were also reported to be low in this group. In the study, ERCC2, ATM, FANCC and RB1 gene mutations that may be related to neoadjuvant $C T$ hve been also examined. Especially, the ERCC2 gene has been found to be lower in the secondary MIBC group (11\% vs $1.8 \%, p=0.044$ ). Also, while KMT2D change was more frequent in primary MIBC ( $23 \%$ vs $10 \%, \mathrm{p}=0.013)$, STAG2 $(7.8 \%$ vs $17 \%, \mathrm{p}=0.014)$ and TSC1 (5.5\% vs 14\%, $\mathrm{p}=0.007$ ) changes were more frequent in secondary MIBC (18). In the light of all this information, we see that secondary MIBC behaves differently from primary 
MIBC both molecularly and oncologically. Therefore, besides the standard approach, disease-specific approaches should be developed for this patient group. When we examine the points that need to be emphasized, neoadjuvant $\mathrm{CT}$ and early RC in secondary MIBC should be investigated in large series, new agents as neoadjuvant options should be investigated and RC option should be discussed in the high risk NMIBC group.

\section{Study Limitations}

Considering the limitations of our study, the low number of all patients in the study and low number of patients in the secondary MIBC group were observed as important limitations. Our secondary MIBC rate (18.7\%) was found to be lower than retrospective large series $(31.7-43 \%)(7,9,10,18,19)$. However, our rate was observed to be similar to prospective series (14.9$23.4 \%)(11,17,18)$. Although the number of patients was small, the fact that patients with complete data were examined in our study, partially reduced the possibility of bias. Therefore, although no significant difference was observed in terms of the oncological results, the existence of significant survival differences between the groups showed that our results could be taken into account.

\section{Conclusion}

Pathological T stage after RC was similar in primary and secondary MIBC was similar, lymph node positivity was lower, mortality was higher, overall survival and cancer specific survival were lower in secondary MIBC statistically. This situation shows us that we should be careful in patient management both before and after the development of muscle invasion in patients with secondary MIBC and that early RC is an important option. Nevertheless, the findings of our study should be examined in larger series and with molecular mechanisms.

\section{Acknowledgements}

Publication: This study was presented in the $14^{\text {th }}$ International Urooncology Congress on November 6-10 in 2019, Antalya.

Contribution: There is not any contributors who may not be listed as authors.

Conflict of Interest: No conflict of interest was declared by the authors.

Financial Disclosure: The authors declared that this study received no financial support.

\section{Ethics}

Ethics Committee Approval: This study is retrospective.

Informed Consent: This study is retrospective.

Peer-review: Externally and internally peer-reviwed.

\section{Authorship Contributions}

Concept: A.E., B.G., T.D., Design: S.Ç., Data Collection and Processing: I.H.B., Analysis and Interpretation: E.Ş., S.Ç.,
Literature Search: I.B., Writing: S.Ç., A.E.

\section{References}

1. Siegel RL, Miller KD, Jemal A. Cancer Statistics, 2019. CA Cancer J Clin 2019;69:7-34.

2. Albano JD, Ward E, Jemal A, et al. Cancer mortality in the United States by education level and race. I Natl Cancer Inst 2007;99:1384-1394.

3. Hidas G, Pode D, Shapiro A, et al. The natural history of secondary muscle-invasive bladder cancer. BMC Urol 2013;13:23.

4. Breau RH, Karnes RJ, Farmer SA, et al. Progression to detrusor muscle invasion during urothelial carcinoma surveillance is associated with poor prognosis. BJUI 2014;113:900-906.

5. Chamie K, Litwin MS, Bassett JC, et al. Recurrence of high-risk bladder cancer: a population-based analysis. Cancer 2013;119:3219-3227.

6. Babjuk M, Burger M, Compérat E, et al. EAU Guidelines on Nonmuscle-invasive Bladder Cancer 2019. https://uroweb.org/guideline/ non-muscle-invasive-bladder-cancer/

7. Ge P, Wang L, Lu M, et al. Oncological Outcome of Primary and Secondary Muscle-Invasive Bladder Cancer: A Systematic Review and Meta-analysis. Sci Rep 2018;8:7543.

8. Witjes JA, Bruins HM, Cathomas R, et al. EAU Guidelines on Muscleinvasive and Metastatic Bladder Cancer. 2019. https://uroweb.org/ guideline/bladder-cancer-muscle-invasive-and-metastatic/

9. Vaidya A, Soloway MS, Hawke C, et al. De novo muscle invasive bladder cancer: is there a change in trend? J Urol 2001;165:47-50.

10. Moschini M, Sharma V, Dell'oglio $P$, et al. Comparing long-term outcomes of primary and progressive carcinoma invading bladder muscle after radical cystectomy. BJUI 2016;117:604-610.

11. Aziz A, Gierth M, Fritsche HM, et al. Oncological outcome of primary versus secondary muscle-invasive bladder cancer is comparable after radical cystectomy. Urol Int 2013;91:97-102.

12. Sylvester RJ, van der Meijden APM, Oosterlinck W, et al. Predicting recurrence and progression in individual patients with stage Ta T1 bladder cancer using EORTC risk tables: a combined analysis of 2596 patients from seven EORTC trials. Eur Urol 2006;49:465-466.

13. Herr HW, Donat SM. A re-staging transurethral resection predicts early progression of superficial bladder cancer. BJUI 2006;97:1194-1198.

14. Gore JL, Lai J, Setodji CM, et al. Mortality increases when radical cystectomy is delayed more than 12 weeks: results from a Surveillance, Epidemiology, and End Results-Medicare analysis. Cancer 2009;115:988-996.

15. Lee CT, Madii R, Daignault S, et al. Cystectomy delay more than 3 months from initial bladder cancer diagnosis results in decreased disease specific and overall survival. J Urol 2006;175:1262-1267.

16. Fahmy NM, Mahmud S, Aprikian AG. Delay in the surgical treatment of bladder cancer and survival: systematic review of the literature. Eur Urol 2006;50:1176-1182.

17. May M, Burger M, Brookman-May S, et al. EORTC progression score identifies patients at high risk of cancer-specific mortality after radical cystectomy for secondary muscle-invasive bladder cancer. Clin Genitourin Cancer 2014;12:278-286.

18. Pietzak EJ, Zabor EC, Bagrodia A, et al. Genomic Differences Between "Primary" and "Secondary" Muscle-invasive Bladder Cancer as a Basis for Disparate Outcomes to Cisplatin-based Neoadjuvant Chemotherapy. Eur Urol 2019;75:231-239.

19. Kotb AF, Kovac E, Kassouf W, et al. Radical cystectomy for clinically muscle invasive bladder cancer: does prior non-invasive disease affect clinical outcomes? World J Urol 2012;30:761-767. 